\section{THE UNIVERSITY OF WESTERN} ONTARIO MEDICAL JOURNAL

Volume 84, Issue 1, Winter 2015 www.uwomj.com

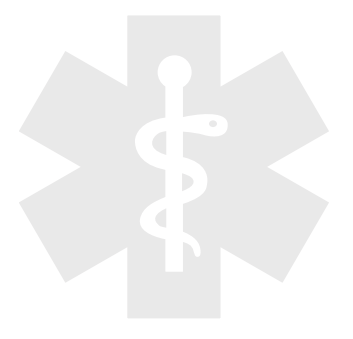

\section{EDITORIAL}

3 Mysteries of the brain and mind Jason L. Chan

\section{FEATURE ARTICLE}

4 Care and curriculum: does clinical empathy decline during medical education? Victor Parchment (BA Candidate), Naomi Mudachi (Meds 2017)

Faculty Reviewer: Dr Barry Schwartz, DDS, MHSc (Division of Practice Administration)

\section{BRAIN AND MIND ARTICLES}

7 Tick tock: thrombolysis \& acute management of ischemic stroke Alexander Levit (MD/PhD 2020), Brandon Chau (Meds 2018)

Faculty Reviewer: Dr Vladimir Hachinski, CM, MD, DSc, FRCPC (Department of Clinical Neurological Sciences)

10 A brief review of neuroimaging using functional magnetic resonance imaging (fMRI) Stefan Rodic (Meds 2018), Pei Jun Zhao (Meds 2017)

Faculty Reviewer: Dr Ravi Menon, PhD (Department of Medical Biophysics)

13 Cognitive liberty: protecting the right to neuroenhancement Arthur Shuster (Meds 2017), Adriana Cappelletti (Meds 2018)

Faculty Reviewer: Dr Jackie Sullivan, PhD (Department of Philosophy)

15 Physician compensation structures and how they incentivize specific patient care behaviour Michael Hewak (Meds 2016), Adam Kovacs-Litman (Meds 2018)

Faculty Reviewer: Dr Javeed Sukhera, MD, FRCPC (Department of Psychiatry)

18 What kills us and what costs us: an examination of the ALS Ice Bucket Challenge Keegan Guidolin (Meds 2017), Matthew Douglas-Vail (Meds 2018)

Faculty Reviewer: Dr Shannon L Venance, MD, PhD, FRCPC (Department of Clinical Neurological Sciences)

20 Separation and reunion: a short history of mind-body dualism Stephanie Mokrycke (Meds 2017), Hao Li (Meds 2016)

Faculty Reviewer: Dr Shelley McKellar, PhD (Department of History)

23 A day with an orthoptist Jeffrey Law (Meds 2016), Charles Yin (MD/PhD 2021)

Reviewer: Charla Snow, BSc, OC(C)

25 Optogenetics: illuminating the brain Phillip Williams (Meds 2017), Steven Wong (Meds 2018)

Faculty Reviewer: Dr Susanne Schmid, PhD (Department of Anatomy and Cell Biology)

29 Conversations with a pediatric psychiatrist

Han Yan (Meds 2017), Ramona Neferu (Meds 2018)

Faculty Reviewer: Dr Javeed Sukhera, MD, FRCPC (Department of Psychiatry),

31 Conversations with a neurologist Han Yan (Meds 2017), Ramona Neferu (Meds 2018)

Faculty Reviewer: Dr Christen Shoesmith, MD, FRCPC (Department of Clinical Neurological Sciences)

33 Conversations with a neurosurgeon

Han Yan (Meds 2017), Ramona Neferu (Meds 2018)

Faculty Reviewer: Dr Fawaz Siddiqi, MD, MBA, FRCSC, FACS (Department of Clinical Neurological Sciences)

35 When the beat clots: ischemic stroke in atrial fibrillation Kevin Braden (Meds 2017), Nicole Arseneau (Meds 2018)

Faculty Reviewer: Dr Allan Skanes, MD, FRCPC (Division of Cardiology)

38 Maggots on the brain (and in it): a case of cerebral myiasis Craig Olmstead (Meds 2017), Charles Jian (Meds 2018)

Faculty Reviewer: Dr Michael John, MD, FRCPC (Division of Infectious Diseases) 
EXECUTIVE TEAM

EDITOR IN CHIEF

Jason Chan (MD/PhD 2017)

SENIOR ASSOCIATE EDITOR Anthony Chow (Meds 2016)

Kevan Lu (Meds 2017)

Craig Olmstead (Meds 2017)

Eric Roszell (Meds 2016)

Hao Li (Meds 2016)

JUNIOR BLOG EDITOR Hao Li (Meds 2016)

SENIOR FINANCIAL OFFICER Omer Dost (Meds 2017)

JUNIOR FINANCIAL OFFICER Stephanie Fong (Meds 2018)

LAYOUT EDITOR Andrew Arifin (Meds 2018)

IT MANAGER

Jun Sunny Yin (Meds 2017)

DEPARTMENTAL EDITORS

\begin{tabular}{ll} 
CLINICAL PROCEDURES & $\begin{array}{l}\text { Alexander Levit (MD/PhD 2020) } \\
\text { Brandon Chau (Meds 2018) }\end{array}$ \\
\hline DIAGNOSTIC REVIEW & $\begin{array}{l}\text { Pei Jun Zhao (Meds 2017) } \\
\text { Stefan Rodic (Meds 2018) }\end{array}$ \\
\hline ETHICS \& LAW & $\begin{array}{l}\text { Arthur Shuster (Meds 2017) } \\
\text { Adriana Cappelletti (Meds 2018) }\end{array}$ \\
\hline HEALTH POLICY \& ECONOMICS & $\begin{array}{l}\text { Michael Hewak (Meds 2016) } \\
\text { Adam Kovacs-Litman (Meds 2018) }\end{array}$ \\
\hline HEALTH PROMOTION & Keegan Guidolin (Meds 2017) \\
\hline HISTORY OF MEDICINE & Matt Douglas-Vail (Meds 2018) \\
\hline Hao Li (Meds 2016) & Stephanie Mokrycke (Meds 2017) \\
\hline ZEBRA FILES & Jeffrey Law (Meds 2016) \\
\hline Charles Yin (MD/PhD 2021) \\
\hline PROFILES & Phil Williams (Meds 2017) \\
\hline Steven Wong (Meds 2018)
\end{tabular}

THE UNIVERSITY OF WESTERN ONTARIO MEDICAL JOURNAL

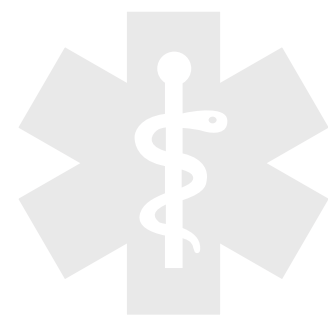

\section{EDITORIAL BOARD}

1 Dr. Lois Champion

2 Dr. Michael Rieder

3 Dr. Jeffrey Nisker

4 Dr. Faisal Rehman

5 Dr. Jim Silcox

6 Dr. Douglas Quan

Cover Art: Han Yan, Meds 2017

Description: A technique developed in 2007 and adapted here in chalk, "Brainbow" maps individual neurons simultaneously with fluorescent proteins. This method randomly expresses different ratios of red, green and blue derivatives, allowing for more than 100 different neurons and their connections to be illuminated. 